Marina lakushevich

\title{
„Immer mehr Menschen fallen in Depressionen“. Mediale Konstruktionen einer Volkskrankheit
}

\begin{abstract}
Der Beitrag widmet sich der medialen Konstruktion des Krankheitsbildes der Depression in den deutschen Qualitätsmedien im Zeitraum 1954-2015. Im Fokus diskurslinguistischer Analysen stehen Diskurshandlungen verschiedener Diskursakteure und ihre Rolle bei der Konstitution des Krankheitsbildes der Depression. Am Beispiel einer grammatischen Konstruktion, ihrer Kontextualisierung und der diskursspezifischen Metaphorik werden Veränderungen in den diskursiven Strategien (Narration und Personalisierung) nachvollzogen.
\end{abstract}

Keywords: Depression, massenmediale Diskurse, Diskursanalyse, Diskursgrammatik, Konstruktion, Narration, Personalisierung

\section{Mediale Realitätskonstruktion}

Die Annahme, dass in den Massenmedien Realität konstruiert wird, wird in der Forschung seit den 1960er Jahren thematisiert (vgl. z. B. Gieber 1964; Galtung \& Ruge 1965; Rosengren 1970; Schulz 1976). Die globale Frage, um die sich diese Untersuchungen drehen, ist das Verhältnis zwischen der „faktischen Realität“ und der von den Medien vermittelten (vgl. Schulz 1976: 25). Allerdings zeigen die Studien, dass es unmöglich ist, seriöse wissenschaftliche Aussagen darüber zu treffen, ob in den Medien die faktische Realität überhaupt dargestellt werden kann. Schulz (1976) schlägt daher vor, die Medienrealität zu untersuchen, also die Art und Weise, wie etwas in den Medien dargestellt wird.

Luhmann $(1975,1988,1995)$ geht noch weiter, indem er die Idee des operativen Konstruktivismus und der sozialen Realitätskonstruktion herausarbeitet: Die Realität ist in den kognitiven Operationen und nicht in der äußeren Welt verortet (vgl. Luhmann 1995: 9). Es gibt nicht die eine Welt „da draußen“ (Luhmann 1988: 16), sondern verschiedene „Wirklichkeiten“ (Luhmann 1995: 18), die auch die gesellschaftliche Kommunikation bedingen. Da die gesellschaftliche Kommunikation $\mathrm{u}$. a. in den massenmedialen Diskursen realisiert wird, ist

Marina Iakushevich, Universität Innsbruck, Institut für Germanistik, Marina.lakushevich@uibk. ac.at

Ә Open Access. ( 2021 Marina lakushevich, publiziert von De Gruyter. (c) BY-NC-ND Dieses Werk ist lizenziert unter einer Creative Commons Namensnennung - Nicht-kommerziell - Keine Bearbeitung 4.0 International Lizenz. https://doi.org/10.1515/9783110688696-023 
es folgerichtig, dass der massenmediale Sprachgebrauch eine gesellschaftliche Domäne darstellt, in der Wirklichkeiten konstituiert werden. Die linguistische Diskursanalyse bietet hier mit ihren theoretischen Annahmen - auch zu der gesellschaftlichen Konstruktion der Realität (vgl. z. B. Spitzmüller \& Warnke 2011: 52-64; Felder 2010: 17-18; Teubert 2019) - und dem methodischen Analyseinventar einen geeigneten Zugang zu den öffentlichen Diskursen.

\section{Linguistische Diskursanalyse}

Unter Diskurs verstehe ich - in Anlehnung an Foucault (1991, 1997) und für die germanistische Linguistik an Busse \& Teubert (1994), Warnke (2009), Felder (2010), Spieß (2011), Spitzmüller \& Warnke (2011) - ein thematisch, semantisch und zeitlich verbundenes Textgeflecht. Foucaults Überlegungen zum Diskurs betreffen allerdings die Sprache nur am Rande, obwohl er die sprachliche Materialisierung der Diskurse erwähnt: „[...] der Diskurs [ist] in seiner materiellen Wirklichkeit [...] gesprochenes oder geschriebenes Ding [...]“ (Foucault 1991: 10). Erst die sprachwissenschaftliche Auseinandersetzung mit den Diskursen (so z. B. der programmatische Aufsatz von Busse \& Teubert 1994) misst der sprachlichen Oberfläche eine wesentliche Bedeutung zu. Die sprachliche Oberfläche ist die Basis der Analysen, die diskursive Strukturen sichtbar werden lassen. Foucault spricht in diesem Zusammenhang von der Herausbildung der Gegenstände durch die Diskurse. Die Diskurse sind „[...] als Praktiken zu behandeln, die systematisch die Gegenstände bilden, von denen sie sprechen“ (Foucault 1997: 74).

Die sprachliche Oberfläche ermöglicht also Zugänge zu Diskursen. Dabei wird unter der linguistischen Perspektive der Zusammenhang zwischen Sprache, Wissen und Gesellschaft fokussiert (vgl. Spitzmüller \& Warnke 2011: 40-48, 52-64). Im Diskurs wird Wissen durch (sprachliche) Handlungen der Diskursakteure konstituiert, die in komplexe soziale Strukturen eingebunden sind: „Diskurse sind gesellschaftliche Praktiken, die sich sprachlich manifestieren und außersprachlich bedingt sind“ (Spieß 2011: 125). Indem die Diskursakteure (sprachliche) Handlungen vollziehen, versuchen sie, Faktizität herzustellen und in den argumentativen Auseinandersetzungen ihre Geltungsansprüche durchzusetzen (vgl. Spitzmüller \& Warnke 2011: 46-47; Felder 2013: 13-16). Die sprachlichen Handlungen von Diskursakteuren lassen sich nach folgenden Handlungstypen klassifizieren und analysieren: 1) Sachverhaltskonstituierung, 2) Sachverhaltsverknüpfung, 3) Sachverhaltsbewertung (vgl. Felder 2013: 118).

Die Diskursakteure treten in bestimmten sozialen Rollen auf: In den sprachlichen Handlungen kontextualisieren sie sprachliche Phänomene und konstituie- 
ren dabei Wissen. Spitzmüller \& Warnke sprechen den Diskursakteuren den Status eines Scharniers zwischen den Einzeltexten und der transtextuellen Ebene zu (vgl. Spitzmüller \& Warnke 2011: 136-137). Dabei greifen sie auf Foucaults Überlegungen zurück, der eine grundsätzliche Frage formuliert hat: „Wer spricht?“ (Foucault 1997: 75). Auch Foucault thematisiert bereits den Status von Sprachinhabern, der ihnen ermöglicht, Diskurse zu gestalten (vgl. Foucault 1997: 75-76). Hierbei sind z. B. die Praktiken der Ausschließung zu nennen, bei denen bestimmte Themen im Laufe der Zeit zuerst mit einem Sprechverbot belegt sind, das sich dann kontinuierlich transformiert und in anderen Praktiken wiederfindet (vgl. Foucault 1991: 39 zu Sexualität).

Unter der Annahme einer generellen Perspektivität von Sprache (vgl. Köller 2004) verweist Felder (2013: 16-17) auf „Denkungsarten und Denkweisen“, die von sprachlichen Phänomenen indiziert werden: Durch den spezifischen Sprachgebrauch von Diskursakteuren würden bestimmte Sichtweisen auf Sachverhalte festgelegt und Wissensrahmen abgesteckt (Felder 2010: 13-14). Dadurch werden Sachverhalte auf spezifische Weisen konstituiert, bewertet und mit anderen Sachverhalten verknüpft. Dies kann in diskursiven Auseinandersetzungen zur Folge haben, dass sich bestimmte, interessengeleitete Sichtweisen durchsetzen. Da Diskurse sich in der Öffentlichkeit manifestieren (vgl. Busse 1996: 347; Spieß 2011: 129), sind Massenmedien also der Ort, an dem Diskurse sichtbar werden. Massenmediale Texte sind daher, nicht zuletzt wegen ihrer Mehrfachadressierung (Kühn 1995), für diskurslinguistische Untersuchungen besonders geeignet, auch weil in den massenmedialen Diskursen gesellschaftlich relevante Themen gesetzt, verhandelt und verbreitet werden (vgl. van Dijk 2009).

\section{Diskursthema und Korpuszusammenstellung}

\subsection{Mediale Medizindiskurse: Depression}

Die Depression zählt zu den häufigsten Krankheiten: Laut WHO sind aktuell (Stand März 2020) mehr als 264 Millionen Menschen weltweit von der Depression betroffen. ${ }^{1}$ Das deutsche Bundesministerium für Gesundheit schätzte 2019, mit Verweis auf die WHO, dass im Jahr 2020 Depressionen und affektive Störungen die zweithäufigste Volkskrankheit werden. ${ }^{2}$ Abgesehen von persönlichem Leid,

$1 \mathrm{https}$ //www.who.int/en/news-room/fact-sheets/detail/depression, letzter Zugriff 07.04.2020. 2 http://www.bundesgesundheitsministerium.de/themen/praevention/gesundheitsgefahren/ depression.html, letzter Zugriff 07.04.2020. 
Beeinträchtigungen im alltäglichen Leben, die die an einer Depression Erkrankten erfahren, und im schlimmsten Fall einem Suizid, stellen Volkskrankheiten einen nicht unerheblichen wirtschaftlichen Faktor dar. Sie verursachen Krankschreibungen, verminderte Produktivität, Berufsunfähigkeit. Laut Berichten der deutschen Krankenkassen stehen psychische Erkrankungen bei den Frühberentungen an erster Stelle; dabei dominieren bei affektiven Störungen die Depressionen. ${ }^{3}$

So ist es verständlich, dass Krankheiten, als gesellschaftlich relevante Inhalte, in den medialen Diskursen ständig präsent sind. Die Gesundheit wird nicht nur als ein Wirtschaftsfaktor, sondern als ein hohes privates und gesellschaftliches Gut betrachtet. Die ständige Präsenz von medizinischen Themen veranlasst Busch, von „Medikalisierung“ der öffentlichen Diskurse zu sprechen (Busch 2015: 369). Kickbusch beobachtet ein „signifikantes Ansteigen des öffentlichen und persönlichen Interesses an Gesundheit“ (Kickbusch 2006: 17), das zu einer allgemeinen Durchdringung des gesellschaftlichen Diskurses mit Fragen zu Gesundheit und Krankheit führt (vgl. Kohring 2005: 27-28). Mediziner*innen beklagen Medikalisierungs- und Pathologisierungsprozesse in der öffentlichen Diskussion (vgl. Schneider \& Strauß 2013: 217).

Unter Berücksichtigung der oben diskutierten Aspekte wurde das Analysekorpus nach folgenden Kriterien zusammengestellt. In den Archiven der deutschen Qualitätszeitungen DIE ZEIT, DER SPIEGEL, FAZ wurden nach dem Stichwort „Depression“ Texte recherchiert. Ausgewählt wurden 696 Texte im Zeitraum 1954-2015, die die Krankheit Depression schwerpunktmäßig thematisieren, d. h. die Krankheitsdarstellungen mit Symptomen und Therapien. ${ }^{4}$ Die Konzentration auf die Depressionssymptome in den Texten kann dadurch erklärt werden, dass auch die Fachdefinitionen von psychischen Krankheiten symptombasiert sind (hier vor allem ICD und DSM). ${ }^{5}$ In den 1950ern und 1960ern tauchen nur

3 Diese Angaben sind der Broschüre des Robert Koch Instituts Heft 51 Depressive Erkrankungen (2010) entnommen (https://www.rki.de/DE/Content/Gesundheitsmonitoring/Gesundheits berichterstattung/GBEDownloadsT/depression.html;jsessionid=53155C7E4C722B6804866485 6B3BB3BD.internet072?nn=2370692, letzter Zugriff 07.04.2020).

4 Die Verteilung nach den einzelnen Medien und Jahrabschnitten ist folgende: DIE ZEIT: 261 Texte, DER SPIEGEL: 105 Texte und FAZ 330 Texte.

5 ICD steht für International Statistical Classification of Diseases and Related Health Problems, die aktuell in der 10. Version von 2019 vorliegt, ICD-10, für Deutschland ICD-10-GM, (https://www.dimdi.de/static/de/klassifikationen/icd/icd-10-gm/kode-suche/htmlgm2019/ letzter Zugriff 9.04.2020); DSM ist Diagnostisches und statistisches Manual psychischer Störungen, das von der Amerikanischen Psychiatrischen Assoziation herausgegeben wird und aktuell in der 5. Version vorliegt, DSM-5, (https://www.psychiatry.org/psychiatrists/practice/ dsm, letzter Zugriff 9.04.2020). 
einzelne Texte zu Depression auf, sieben Texte in der ZEIT, zwei Texte im SPIEGEL. Seit den 1990er Jahren wächst die Anzahl der Texte, nicht zuletzt dank den Online-Ausgaben: So sind z. B. in der FAZ in den 1990ern dreizehn Texte erscheinen, seit 2000 197. Die Texte sind in den Ressorts Wissen, Wissenschaft erschienen: Im Vordergrund der journalistischen Berichtserstattung steht Wissensvermittlung. ${ }^{6}$ Die nachfolgenden Analysen dienen der Beantwortung der in diesem Beitrag relevanten Forschungsfrage nach dem massenmedial konstituierten Krankheitsbild der Depression (im Sinne einer Sachverhaltskonstituierung nach Felder 2013: 118) und dessen Veränderungen im untersuchten Zeitraum. Dabei geht es vorrangig um die Darstellung der Krankheitssymptome aus der Perspektive verschiedener Diskursakteure. Das konkrete methodische Vorgehen besteht in der Analyse einer grammatischen Konstruktion und deren diskursiven Funktionen.

\subsection{Diskursgrammatik}

Die diskurslinguistische Annahme über den Zusammenhang von Sprache, Wissen und Gesellschaft kann in konkreten Analysen heißen, dass man indexikalische Beziehungen zwischen den sprachlichen Zeichen und gesellschaftlich relevanten Aspekten ihres Gebrauchs offenlegt. So postuliert Müller, dass „,...] sprachliche Zeichen kraft der Tatsache, dass sie in sozialen Zusammenhängen stehen, in indexikalischen Ordnungen ihres Gebrauchs zu verorten sind und diese gleichzeitig hervorbringen“ (Müller 2016: 303). Seriell auftretende sprachliche Ausdrücke können als Spuren sozialer Interaktion gesehen werden: Ihr Gebrauch ist durch situative, soziale, aber auch historische und politische Parameter determiniert und wirkt auf sie zurück.

Im Folgenden konzentrieren sich die Analysen auf eine grammatische Konstruktion, die für die narrative Struktur der untersuchten Texte wesentlich ist, da sie die Komplikation (dazu weiter unten) (Labov \& Waletzki 1967: 32) markiert. ${ }^{7}$ Unter Konstruktion verstehe ich im Sinne der kognitiv-funktional orientierten Konstruktionsgrammatik „[...] PAIRINGS OF FORM WITH SEMANTIC OR DISCOURSE FUNCTION, including morphemes or words, idioms, partially lexically filled or fully general phrasal patterns“ (Goldberg 2006: 5, Hervorhebung

\footnotetext{
6 Die in der Medienwissenschaft und Publizistik etablierte Ansicht über den Wissenschaftsjournalismus als Berichtserstattung aus der Wissenschaft in die Gesellschaft (vgl. Kohring 2005: 9) kann durchaus kritisch betrachtet werden, im Rahmen dieses Beitrags wird nur kurz darauf eingegangen.

7 Die Wahl der Konstruktion ist den diskurssemantischen Überlegungen geschuldet, also corpus based (vgl. Spitzmüller \& Warnke 2011: 39).
} 
im Original). Die wesentliche Überlegung für diese Ausrichtung der Konstruktionsgrammatik war die Berücksichtigung des konkreten und kontextgebundenen Sprachgebrauchs, was gerade für diskurslinguistische Analysen sehr brauchbar ist. In der Tradition der kognitiv-funktional orientierten Konstruktionsgrammatik werden grammatische Muster analysiert als „[...] komplexe Konstellationen charakteristischer formaler und funktionaler Eigenschaften [...], die sich im situativen Sprachgebrauch als rekurrente Lösungen für bestimmte kommunikative Problem- und Aufgabenbereiche herausgebildet haben“ (Bücker, Günthner \& Imo 2015: 2). In den folgenden Analysen wird zu zeigen sein, welche konkreten konstruktionalen Eigenschaften auf der textuellen und diskursiven Ebene realisiert werden.

\section{$4\left[[i n]\left[D_{\text {akk }}\right][\right.$ Depression][V]]: Konstruktion und Diskurs}

Die konkreten Realisierungen der Konstruktion [[in][DET ${ }_{\mathrm{akk}}[\text { Depression] }[\mathrm{V}]]^{8}$ wurden mit AntConc (Antony 2014) extrahiert. ${ }^{9}$ Dabei zeigt sich, dass sich die lexikalische Ausfüllung des Verbalslots [V] und der direktive Präpositionalgebrauch in der Präpositionalphrase gegenseitig bedingen. Der verbale Slot wird von den Verben fallen, verfallen, stürzen, rutschen, geraten besetzt, wie in den folgenden Beispielen deutlich wird:

1) Ich rutschte zurück in die Depression, und wie bei den meisten verlief dieser Prozess so schleichend, dass ich es nicht merkte. (SPIEGEL-ONLINE 17.11.2009)

2) Während manche Patienten rasche, unvorhersehbare Umschwünge ihrer Stimmung erfahren, erleben andere vornehmlich die Höhenflüge der Manie oder stürzen regelmäßig in Depressionen. (FAZ, 21.02.2007, S. N2)

3) [...] oder ob sich im Oktober 1994 im kanadischen Langley drei halbwüchsige junge Männer umbrachten, indem sie Auspuffgase in ihr Auto leitetenin - in ihrem Abschiedsbrief hieß es, sie seien nach dem Tod des Rocksängers Kurt Cobain „in Depressionen verfallen“. (DER SPIEGEL 26.06.1995, S. 168)

Wie man anhand dieser Beispiele sehen kann, werden mit der Konstruktion subjektive, persönliche Erfahrungen der betroffenen Menschen versprachlicht.

8 Die Schreibweise entspricht den konstruktionsgrammatischen Konventionen.

9 Eine ausführliche Diskussion zum Status dieser Konstruktion s. Iakushevich (2020b). 
Durch die Verben fallen, verfallen, stürzen, rutschen, geraten wird das Erkranken an einer Depression als ein ungeplanter, ungewollter und unkontrollierter Vorfall konzeptualisiert. Dieser Moment wird von den Menschen als ein Moment des absoluten Kontrollverlusts erlebt, ähnlich wie wenn man z. B. plötzlich ausrutscht und hinfällt. Die psychische Krankheit wird zu einer elementaren körperlichen Erfahrung, sie wird erlebt als ein plötzlicher, unvorhergesehener Wechsel von einer normalen Körperposition während der aufrechten Fortbewegung zu einer außergewöhnlichen Körperposition, nämlich auf dem Boden liegend. Vor dem Hintergrund der kognitiven Semantiktheorien kann man in diesen Beispielen die Bestätigung der These von Lakoff \& Johnson sehen, dass kognitive und sprachliche Strukturen in den sensomotorischen Erfahrungen der Menschen verankert sind (Lakoff \& Johnson 1980; vgl. Stöckl 2004: 73-79). Weitere synonyme Ausdrücke, mit denen diese Erfahrungen dargestellt werden, sind ebenfalls konsistent mit der Konzeptualisierung der Depression als Fall, Rutsch, Sturz: z. B. in ein tiefes, schwarzes Loch fallen.

Die Semantik dieser Konstruktionsrealisierungen liefert wichtige Aspekte bezüglich des medialen Krankheitsbilds der Depression. Die Depression wird dargestellt als ein Fall, eine Unterbrechung des gewöhnlichen Lebenslaufs der betroffenen Menschen. In der Semantik der Verben fallen, verfallen, stürzen, rutschen, geraten sowie der der gesamten Konstruktion ist der Aspekt der Temporalität ausgedrückt. Wenn auch fallen und geraten eine plötzliche, sehr schnelle Bewegung implizieren und rutschen eine eher langsame und länger andauernde, bezeichnen doch alle Verben eine Bewegung, die die Zustandsveränderung genau beschreiben.

Die Bedeutung der einzelnen Konstruktionselemente, der Präpositionalphrase, der Verbalphrase, aber auch der einzelnen Wörter in, Depression, fallen, verfallen, stürzen, rutschen ist in den untersuchten konkreten Gebrauchskontexten metaphorisch. Depression geht auf das lateinische depprimere (herabdrücken, -senken) zurück (vgl. Pfeifer 2012: 215). Die direktionale Verwendung der Präposition in in den untersuchten Konstruktionsrealisierungen schließt eine rein physikalische Richtungsbezeichnung aus (vgl. Zifonun, Hoffmann \& Strecker 1997: 2128), dasselbe gilt auch für die Verben. Auch die Gesamtbedeutung des Ausdrucks ist metaphorisch. Interessanterweise gibt es aber für diese metaphorische Bedeutung in dem hier relevanten Kontext keine wörtliche bzw. nicht metaphorische Entsprechung. Die Depression wird als ein Raum konzeptualisiert, in den man wider Willen und unvorbereitet gerät. Auf die gleiche Weise werden im Deutschen z. B. Emotionen konzeptualisiert und ebenfalls mit lokalen und direktiven Präpositionen ausgedrückt, z. B. in Rage, in Wut, in Stimmung (vgl. Zifonun, Hoffmann \& Strecker 1997: 2128). Eine Depression dürfte aber natürlich viel komplexer sein als eine Emotion. 
Das Erleben der Depression als ein plötzlicher Fall, Sturz, Rutsch verweist auf das Schicksalhafte dieser Krankheitserfahrung: Es ist nichts, was man durch eigenes schuldhaftes Handeln verursacht. Die Depression widerfährt einem, stößt einem $\mathrm{zu}^{10}{ }^{10}$ Implizit wird damit die Frage nach einer persönlichen Schuld aufgeworfen. Was hat man falsch gemacht? Was hat zu der Erkrankung geführt? Im folgenden Beispiel ist diese Implizitheit im Ausdruck nichteingestandene Affekte sichtbar:

4) Bedrohlich wird die Verdrängung nur, wenn nichteingestandene Affekte in Depression oder Aggression führen, wenn der Betroffene mit der Verleugnung nicht leben kann. (ZEIT ONLINE, 05.02.1998)

Durch die Konzeptualisierung der Depression als Schicksal wird die Frage nach der persönlichen Schuld zurückgewiesen, die Depression kann jederzeit jeden treffen.

In der Darstellung als ein Schicksalsschlag, als etwas, was plötzlich, unerwartet und ungeplant in das Leben der Menschen tritt, wird die Depression als ein totaler Kontrollverlust über die eigenen Gefühle, Gedanken und Handlungen erlebt, als Verlust des Selbst, als etwas, was das gesamte Ich, die gesamte Persönlichkeit betrifft. ${ }^{11}$ Bei den Menschen mit einer Depression wird eine geringe Agency vorausgesetzt, darunter wird die subjektive Überzeugung über die eigene Wirkmächtigkeit verstanden (vgl. Bandura 2000). ${ }^{12}$ Während einer Depression fühlt man sich schwach, tatenlos, ausgeliefert, handlungsunfähig und umso aktiver erscheint die Krankheit. Die Depression wird personalisiert und als Feind, Aggressor, ein feindliches Gegenüber erlebt. Die betroffene Person sieht sich in einem Kampf mit einem Gegner, den man wie in einem Krieg bekämpfen und besiegen muss, sonst ist man selbst ein Verlierer und Unterlegener, wie unten im Beispiel 5 dargestellt. Damit wird die Metapher KRANKHEIT IST FEIND realisiert, bei der insbesondere der Aspekt des individuellen Kampfes, quasi Mann gegen Mann, zum Tragen kommt. Diese Metapher ist konsistent mit der Metapher KRANKHEIT IST KRIEG, die im Kontext vieler Krankheiten gebraucht wird. ${ }^{13}$

10 Syntaktisch ließe sich die Konstruktion nach Prädikatstypen klassifizieren (vgl. Hartmann 1961: 60), die ich um einen weiteren Typ, das widerfährt-Prädikat, erweitert habe (vgl. Iakushevich 2009: 13-15).

11 Ein bekanntes Werk über Depression des Soziologen Ehrenberg heißt Das erschöpfte Selbst (Ehrenberg 2013).

12 Agency war das Thema einer Datensitzung während des 4. Netzwerktreffens „Linguistik und Medizin“ zum Thema Sprache, Alter, Pflege am 24. Januar 2020 am IDS in Mannheim.

13 Hier sei vor allem Susan Sontags berühmter Essay Krankheit als Metapher erwähnt, in dem sie explizit die Kriegs- und Kampfmetaphorik in Bezug auf Krebs thematisiert: „Es gibt den ,Kampf‘ oder ,Kreuzzug‘ gegen den Krebs, [...]; Menschen, die Krebs haben, sind ,Krebsopfer،.“ 
5) Der 25 Jahre alter Karlsruher gilt als ausgeglichener, eher zurückhaltender Mensch, ein Profi mit vorbildlicher Einstellung. Doch in den vergangenen Monaten ist es ihm schon ein paar Mal passiert, dass er, wenn er im Hotelzimmer sitzt, in ein „tiefes Loch“ fällt. Diese „Durchhänger“ bekämpft er, meist gemeinsam mit seinem Zimmergenossen Carsten Ramelow, mit Computerspielen. (FAZ 274/1999, S 53)

Die Depression als ein aktiv kämpfender Feind wird in den folgenden Beispielen sprachlich vor allem durch die Wahl der Verben konstituiert (meine Hervorhebungen in den Beispielen 6) - 7)):

6) Als Gewinn werten es die New Yorker Psychiater deshalb bereits, wenn das Gemütsleiden überhaupt erkannt wird. Nur so, glauben sie, können Kranke, denen sich die undurchdringlichen Schleier über die Seele gelegt haben, wenigstens vor dem Schlimmsten bewahrt werden. Bei Nachforschungen im Leben von Selbstmordopfern etwa zeigte sich, dass Depressionen die meisten von ihnen bis an die Schwelle des Todes verfolgt hatten. (DER SPIEGEL 38/1989, S. 232-234)

7) 20 Jahre beherrschte sie sein Leben, raubte ihm die Energie, hinderte ihn am Leben. Und demütigte ihn. [...] Fast hätte die Krankheit ihn zerstört - auch weil viele ihm seine Qualen nicht glaubten. (Spiegel-Online, 16.11.2009)

Die Agency zeigt sich in den obigen Beispielen als eine Eigenschaft, die der Depression zugesprochen wird und nicht den Menschen als handelnden Subjekten. Das mediale Krankheitsbild bekommt dadurch weitere Merkmale dazu. Die Darstellung der Depression als Agens und aktiv handelnde Instanz wird, genauso wie im Fall der oben diskutierten Konstruktion, aus der Perspektive der kranken Menschen vorgenommen. In den Beispielen 8) bis 9) werden subjektive Erfahrungen thematisiert; es ist das individuelle Wissen, das hier den Eingang in den Diskurs findet. Es kann von verschiedenen Akteuren in diskursiven Praktiken verhandelt, verbreitet, mit anderen Wissensbeständen verknüpft und zur Durchsetzung von Geltungsansprüchen verwendet werden (vgl. Felder 2013: 14-17).

(Sontag 1980: 62). Aktuell, 2020, bestimmt die Corona-Kriegsmetaphorik die medialen Diskurse in Deutschland und Österreich, nicht zuletzt deshalb, weil auch die medizinische Fachterminologie sich dieser Metaphorik bedient. So spricht man z. B. von „the trajectory and the impact of the COVID-19 epidemic“ (van Damme et al. 2020: 1), ,the most explosive COVID-19 epidemics“ (van Damme et al. 2020: 3). 
Mit den Ausdrücken in eine Depression, fallen, verfallen, stürzen, rutschen werden von den betroffenen Menschen, die in den untersuchten Diskursabschnitten als Diskursakteure fungieren, ihre quälenden, sehr individuellen und fundamentalen Erfahrungen mit der Depression versprachlicht. Gleichzeitig markieren sie einen Lebenseinschnitt, ein wichtiges Ereignis im Leben dieser Menschen. Die Depression wird erlebt als ein Hindernis auf dem Lebensweg, als eine Unterbrechung des Lebenslaufs, als etwas Schicksalhaftes, was den Menschen widerfährt. Solche Darstellungen konzeptualisieren die Krankheit als einen Weg (im Sinne der konzeptuellen Metapherntheorie nach Lakoff \& Johnson 1980). Die Konzeptualisierung der Depression als ein Weg ist relevant zum einen für den massenmedialen Depressionsdiskurs, ${ }^{14}$ zum anderen ist diese Metapher mit einer anderen Metapher konsistent, die in vielen Lebensbereichen allgegenwärtig zu sein scheint: LEBEN IST EIN WEG. ${ }^{15}$

Die Metapher DEPRESSION IST EIN WEG kann im untersuchten Diskursabschnitt als diskurskonstitutiv betrachtet werden (vgl. Spieß 2011, 2014: 46; Hampe 2017). ${ }^{16}$ Unterschiedliche Aspekte der Krankheit konstituieren ein Krankheitsbild, das für den Diskurs als relevant erachtet wird und auf das in den diskursiven Aushandlungsprozessen immer wieder Bezug genommen wird. Auch hier spielt der Aspekt der Temporalität eine wesentliche Rolle. Die Depression wird als ein Prozess dargestellt, der von den Betroffenen erlebt und bewältigt werden muss. Wurde, wie oben dargestellt, der Moment des Erkrankens durch eine grammatische Konstruktion in seiner Punktualität, Plötzlichkeit dargestellt, wird die Krankheit als Prozess vor allem in der textuellen Organisationsstruktur sichtbar. Das Prozessuale, das Dauerhafte der Depression ist durch die Metapher DEPRESSION IST EIN WEG dargestellt, die nicht durch einzelne Lexeme realisiert, sondern durch z. T. mehrere sich anschließende Sätze.

Die nachfolgenden Beispiele zeigen exemplarisch mit Bezug auf einige Temporalmarker (Wortsemantik, Temporaladverbien, Tempus verbi), wie die Diskursmetapher DEPRESSION IST EIN WEG textuell realisiert wird (meine Hervorhebung):

8) Ohne ein Blatt vor den Mund zu nehmen, schildert die Autorin - mit vollem Bekenntnis zur mitunter resignativen Ausgangslage - ihr eigenes Erlebnis

14 Dass nur bestimmte Diskursabschnitte und nicht der gesamte Diskurs zu einem Thema überhaupt untersucht werden können, geht schon aus Foucaults Diskursdefinition hervor, s. Kapitel 2 dieses Beitrags.

15 Die Schreibweise in Großbuchstaben richtet sich nach der üblichen Tradition der kognitiven Metapherntheorien.

16 Die depressionstypische Metaphorik in multimodalen medialen Kontexten habe ich ausführlich an einer anderen Stelle diskutiert (Iakushevich 2020a). 
auf dem Leidensweg einer Depression. Auf ihm begegnete sie mehreren Ärzten, die einen Griff zum Rezeptblock für die ultima ratio hielten. Sie bringt aber auch die hierfür unabdingbare Kraft auf, die freie Arztwahl für sich in Anspruch zu nehmen, ohne in generelle Mediziner-Hetze zu verfallen. (ZEIT Online 11.10.1985)

9) Lange verschwieg sie diese Gedanken. "Ich schämte mich so dafür und habe versucht, mir nichts anmerken zu lassen”, sagt Weimer. Erst sechs Wochen nach der Geburt vertraute sie sich einem Arzt an. [...]

Dann begann ein langer Weg. Gespräche, Selbsthilfegruppen, MutterKind-Kur - es dauerte mehrere Monate, bis es ihr besserging, und ein Jahr, bis die Krankheit geheilt war. "Es muss nicht sein, dass so viele Frauen in diese Depression rutschen - und so lange damit kämpfen", sagt Weimer. Damit manche den Kampf schneller gewinnen können, gründete sie die "Blues Sisters". (FAZ 18.07.2007, S. 40)

Die Beispiele zeigen, wie komplex transtextuelle Diskursanalysen wirklich sind. An der Verwendung der Metapher DEPRESSION IST EIN WEG ist ersichtlich, dass die Analyse von einzelnen Metaphernlexemen, wie Spitzmüller \& Warnke (2011: 201) in dem DIMEAN-Modell vorschlagen, nicht immer zielführend sein kann, da Metaphern oft nicht auf der Ebene der Einzellexeme funktionieren, sondern textuell und diskursiv realisiert werden. ${ }^{17}$ In den analysierten Diskursabschnitten wird die Metapher diskurskonstituierend und -strukturierend verwendet. Dank dieser übergreifenden Metapher entsteht ein spezifisches mediales Bild der Depression, das zum großen Teil auf den subjektiven Erfahrungen Betroffener basiert. In diesen Erfahrungen ist der essentielle Moment des Erkrankens textuell durch eine Konstruktion markiert, die wiederum mit - in diesem medialen Kontext - depressionsspezifischen lexikalischen Füllungen versehen ist.

\section{Diskursstrategien: Narration und Personalisierung}

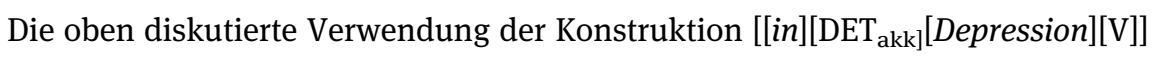
und die depressionstypische Metaphorik - die hier exemplarisch herausgegriffen werden - indizieren spezifische diskursive Strategien.

17 Siehe Hampe (2017: 7) zu "the dynamic multidimensional socio-cognitive model of metaphor". 
Die Konstruktion [[in][⿰DET $\mathrm{akk}_{\text {ak }}[$ Depression][V]] wird in dem untersuchten Diskursabschnitt hauptsächlich in narrativen Strukturen verwendet. Grundlegend für Narration ist „[...] die Zeit als Abfolge von Ereignissen.“ (Vogt 2014: 96). Die Temporalität, die mit verschiedenen Mitteln zum Ausdruck gebracht werden kann, kann also als eine grundlegende Voraussetzung für narrative Strukturen betrachtet werden. Auch für die textuelle Organisation wird die sequenzielle Abfolge von Ereignissen als wesentlich erachtet; Heinemann und Viehweger betonen dies in Bezug auf die chronologische Ordnung von illokutiven Handlungen (vgl. Heinemann \& Viehweger 1991: 238).

Labov und Waletzky haben Narrationen als „oral versions of personal experience“ definiert (Labov \& Waletzky 1967), dabei stellen sie ebenfalls die temporale Abfolge in der Darstellung von persönlichen Erfahrungen (Labov \& Waletzky 1967: 13; vgl. Brinker 2010: 61) in den Mittelpunkt ihrer Überlegungen. Die narrative Struktur besteht nach Labov und Waletzky in der linearen Abfolge von folgenden Kategorien: „orientation“, „complication“, „evaluation“, „resolution“, „coda“ (Labov \& Waletzky 1967: 32-39). ${ }^{18}$ Laut Brinker (2010: 60-61) eignet sich diese Struktur, trotz berechtigter Kritik an ihr, für die Analysen thematischer Grundstrukturen narrativer Texte.

Für die medialen Diskursanalysen sind folgende Elemente brauchbar: der Aspekt der Temporalität, wie bereits oben diskutiert, und die Thematisierung von persönlichen Erfahrungen in den narrativen Kontexten.

Die Konstruktion [[in][DET ${ }_{\text {akk }}[$ Depression][V]] ist in dem untersuchten Korpus in narrative Textmuster eingebunden. Geschildert werden subjektive Erfahrungen der an einer Depression erkrankten Menschen, „complication“ im Sinne von Labov \& Waletzky. In den konkreten mikrotextuellen Kontexten, also der unmittelbaren textuellen Umgebung der Konstruktionsrealisierungen, wird die Subjektposition, meistens in der 1. Person Singular, von den erkrankten Personen eingenommen. Das Subjekt ist aus der Sicht der semantischen Rollen ein Experiens/Erfahrender (vgl. Polenz 1988: 170). Es werden, auch durch Zitate markiert, die persönlichen Geschichten dieser Menschen erzählt. Die Kranken werden somit zu Diskursakteuren, sie bringen eine zusätzliche Perspektive (im Sinne Köller 2004) in den Diskurs, sie bekommen eine Stimme (siehe Foucaults oben diskutierte Frage „Wer spricht?“). Ihre Perspektive ist einerseits individuell und subjektiv, aber auch vom Diskurs geprägt: Die Erfahrungen einer psychischen Krankheit sind nur der kranken Person zugänglich; eine psychische Krankheit ist für Außenstehende nicht notwendigerweise sichtbar und erkenn-

18 Bubenhofer (2018: 373) spricht in seiner korpuslinguistischen Studie zu Geburtsberichten von „narrativen Topoi“. 
bar, wie es z. B. ein gebrochenes Bein ist. Die Art und Weise der Kommunikation über Krankheiten ist aber von gesellschaftlichen Konventionen beeinflusst.

Die Ausdrücke in eine Depression fallen, verfallen, rutschen, geraten, stürzen sind metaphorisch, ${ }^{19}$ wie auch andere, ebenfalls psychiatrische Termini wie z. B. niedergedrückte Stimmung, stimmungsaufhellend. Allerdings gibt es für sie keine nichtmetaphorischen Entsprechungen. Feer (vgl. Feer 1987: 23) bemerkt, dass metaphorisches Sprechen für die abstrakten, höchst subjektiven Erfahrungen psychisch kranker Menschen oft die einzige Möglichkeit darstellt, die innerpsychischen Vorgänge zu versprachlichen und zu kommunizieren. ${ }^{20}$

Die subjektiven Erfahrungen kranker Menschen werden in dem untersuchten Diskursabschnitt vor allem in den narrativen Textstrukturen mit einem spezifischen narrativen Muster gebraucht. Heinemann \& Viehweger definieren es als ein erlebnisorientiertes Strukturmuster (NARR II ERZÄHL-Strukturen) im Unterschied zu einem ergebnisorientierten Strukturmuster (NARR I REFERIER-Strukturen) (vgl. Heinemann \& Viehweger 1991: 238-244). Beim erlebnisorientierten Strukturmuster geht es „[...] um die Kennzeichnung [s]einer Erlebnisperspektive, um die subjektive Charakterisierung der Ereignisse [...]“ (Heinemann \& Viehweger 1991: 242). Dabei spielt die Evaluation eine Rolle: Erzählt wird nur das, was aus der individuellen Perspektive relevant erscheint und nicht zwingend objektiv und überprüfbar sein muss. Im folgenden Beispiel beschreibt der an einer Depression erkrankte Mann, wie es sich für ihn anfühlt, in eine Depression zu fallen. Auffällig ist hier, dass er den Fall als langsam bezeichnet. Damit rückt seine subjektive Erlebnisperspektive in den Vordergrund, im Text auch als Zitat markiert. Die Zeitlichkeit des Erlebnisses wird hier besonders hervorgehoben, da sie aus der individuellen Perspektive als relevant eingeschätzt wird. An diesem Beispiel wird m. E. die besondere Konstellation der Konstruktion, ihrer lexikalischen Füllung in diesem spezifischen Kontext und den narrativen Textstrukturen sichtbar:

10) Schon viermal fiel der stämmige Zweiundsechzigjährige in schwere Depressionen, die zwischen drei Monaten und fast einem Jahr dauerten. In der Regel beginnt eine Depression mit Nachdenklichkeit und abgeschwächten Symptomen. „Man fällt langsam hinein und wacht auch langsam wieder auf. Einmal jedoch verschwanden sie über Nacht“, erinnert er sich. (FAZ, 15.04.2002, S. 49)

19 Im Sinne der kognitiven Metapherntheorie nach Lakoff \& Johnson (1980).

20 Zur Rolle von Metaphern bei der Symptomdarstellung in psychiatrischen Krankenakten s. Schuster (2010: 210-230). 
Bemerkenswert ist außerdem, dass das Auftreten der Konstruktion [[in] $\left[\mathrm{DET}_{\mathrm{akk}}[\mathrm{De}\right.$ pression][V]] in dem untersuchten Diskursabschnitt mit dem Auftreten von narrativen Strukturen zusammenhängt. Der erste Beleg für den Konstruktionsgebrauch stammt aus dem Jahr 1995. Bis in die 1990er Jahre treten kranke Menschen als Diskursakteure nicht auf. Es gibt Berichte über anonyme Patienten*innen bzw. Klienten ${ }^{\star}$ innen, Symptome, Therapien und mögliche Ursachen der Depression. Seit den 1990er Jahren werden immer mehr persönliche Geschichten aus der Perspektive der Betroffenen erzählt. Zunehmend werden die Geschichten personalisiert: Konkrete Personen mit Namen und Bild erzählen selbst über ihre Krankheit, wobei sowohl Personen des öffentlichen Lebens wie z. B. Bundespolitiker und Sportler als auch Privatpersonen vertreten sind. Bis in die 1990er Jahre steht dagegen eine Außenperspektive auf die Depression im Mittelpunkt der medialen Aushandlungen. Zwar wird auch von depressiven Symptomen berichtet, aber aus der Sicht der beobachtenden Ärzt*innen, Therapeut*innen oder Journalist*innen. Die Kranken werden z. B. als „mürrisch-reizbar“ oder „apathisch“ charakterisiert (DER SPIEGEL 51, 1978) oder es wird eine typische Sprechzimmer-Situation beschrieben:

11) Da hockt der Patient“, so schilderte kürzlich das deutsche Medizinal-Magazin „Euromed“ eine typische Sprechzimmer-Situation, „gedrückt im allgemeinen Schlappgefühl, mit 'nem bißchen Traurigkeit unausgesprochener Herkunft; da sagt ihm sein Arzt in zudeckender Weise, wie schön doch die Sonne draußen scheine, und er - der Patient - habe doch so fröhliche Buben.“ (DER SPIEGEL, 04.11.1968, S. 202)

Die kranken Menschen treten im Diskurs nur als Objekte auf, sie sind keine Diskursakteure, ihnen wird keine Rolle zugestanden und kein Rederecht erteilt. Bis in die 1990er Jahre sind an der Depression erkrankte Menschen vom medialen Diskurs ausgeschlossen (vgl. dazu Foucaults (1991: 39) Ausschließungsfunktion). Die subjektive Perspektive der Betroffenen ist zu dieser Zeit noch irrelevant und dadurch nicht vorhanden. Auf der textuellen Ebene äußert sich dies z. B. durch Fehlen von narrativen Strukturen.

Treten die Kranken als Diskursakteure auf, bewirkt das nicht nur Veränderungen in textuellen Mustern, sondern in den Prozessen der medialen Konstituierung $^{21}$ des Krankheitsbildes der Depression. Mit ihren individuellen Erfahrungen, ihrer subjektiven Perspektive kommunizieren die Kranken öffentlich ihre individuellen Wirklichkeiten. Damit erheben sie Geltungsansprüche: Ihr individuelles

21 Zur Unterscheidung von Konstruktion und Konstitution s. Spitzmüller \& Warnke (2011: 43-48). 
Laienwissen wird öffentlich, medial präsent und wahrgenommen. Dieses medizinische Alltagswissen wird diskursiv verarbeitet, da es als relevant eingeschätzt wird (vgl. Felder 2013: 15). Die Kranken werden dank ihrer Aufnahme in den Diskurs aufgewertet, als Diskursakteure konstituieren sie das mediale Krankheitsbild der Depression mit.

Die Wissensbestände der an einer Depression leidenden Menschen werden im medialen Diskurs mit weiteren Wissensbeständen verknüpft, z. B. mit dem Wissen von Fachexpert*innen aus der Medizin, Psychologie und Psychiatrie, Neurologie, Chemie. Dies zeigt sich in bestimmten Textstrukturen, die in Diskursmuster übergehen. Auf der mikrotextuellen Ebene können an die narrativen Elemente argumentative, deskriptive oder explikative (im Sinne von thematischen Entfaltungsstrukturen) angeknüpft werden. Insbesondere seit den 2000er Jahren tritt dieses textuelle Muster auf: Als Einstieg dient eine persönliche Geschichte, die von der betroffenen Person selbst erzählt wird. Die Geschichte konzentriert sich auf die Schilderungen von Depressionssymptomen aus der subjektiven Sicht der/des Kranken. Darauf folgen medizinische Erklärungen zu Depression, es wird nach Krankheitsursachen geforscht, Expertinnen werden interviewt, Therapien und Medikamente vorgestellt. Diese sprachlichen Handlungen werden von verschiedenen Akteuren ausgeführt, dabei können Expertinnen Wirkmechanismen von menschlichen Körperfunktionen oder Medikamente beschreiben und erklären und für eine bestimmte Therapieform plädieren. Dieses textuelle Muster wird immer wieder aufgegriffen und wiederholt. In dem untersuchten Diskursabschnitt lässt es sich in allen drei Medien ab den 2000er Jahren beobachten. Auf der übergeordneten diskursiven Ebene bildet sich dadurch auch ein spezifisches Muster, ein komplexer Zusammenhang zwischen den einzelnen Diskursakteuren und ihren Handlungen, zwischen den einzelnen Wissensbeständen, Themen und Subthemen. Daraus entstehen neue intertextuelle und interdiskursive Verflechtungen zu anderen Themen und Diskursen.

Aus der medienwissenschaftlichen Perspektive wird dabei ein weiterer Aspekt relevant, unter dem dieses diskursive Muster bewertet werden kann. Unterschiedliche Diskursakteure können Personen, Institutionen, Organisationen usw. sein. Die Präsenz von Personen als Diskursakteuren ist zwar in dem untersuchten Diskursabschnitt von Anfang an gegeben, das mediale Krankheitsbild wird aber zuerst vor allem von Expert`innen und Journalistinnen konstituiert. Das Merkmal „Personalisierung“, medienwissenschaftlich definiert als „[...] die zunehmende Orientierung an (prominenten) Personen bei der Vermittlung von Medieninhalten.“ (Bentele, Brosius \& Jarren 2013: 214-215), wird intensiver genutzt. Bis in die 1990er Jahre wird über die Depression berichtet, es werden Krankheitsgeschichten erzählt. Danach, durch die neuen Diskursakteure, wer- 
den Inhalte stärker personalisiert, die Depression wird personalisiert, es werden Krankengeschichten erzählt.

\section{Schlussbetrachtung}

Der Beitrag hat gezeigt, wie das massenmediale Krankheitsbild der Depression konstituiert wird. Dabei wurde der Fokus insbesondere auf die Handlungen verschiedener Diskursakteure gelegt. In dem untersuchten Diskursabschnitt konnten folgende Entwicklungen festgestellt werden: In dem untersuchten Zeitraum 1954-2015 verändert sich die Konstellation der Diskursakteure. Die an einer Depression erkrankten Menschen treten seit den 1990er Jahren als Diskursakteure hinzu und konstituieren mit ihrer individuellen Perspektive und ihren subjektiven Erfahrungen das mediale Bild der Depression mit. Ihr Wissen wird im Diskurs als relevant erachtet und spielt in den diskursiven Sinnaushandlungen eine Rolle. Damit einher gehen weitere mediale Veränderungen wie die zunehmende Personalisierung des Depressionsdiskurses, die sich in den narrativen Strukturen äußert. Krankheitsgeschichten werden zu Krankengeschichten. Die Veränderungen im medialen Diskurs sind Anzeichen (im Sinne indexikalischer Beziehungen zwischen Sprache und Gesellschaft nach Müller 2016) dafür, dass sich die gesellschaftliche Bewertung der Depression, die Relevanz dieser Krankheit nicht nur für einzelne Betroffene, sondern auch für die gesamte Gesellschaft geändert hat.

In dem untersuchten Diskursabschnitt wird Depression als eine Krankheit dargestellt, die als ein totaler Kontrollverlust über das ganze Leben und über das Selbst erfahren wird. Die Depression wird als etwas Schicksalhaftes erlebt, dem man passiv gegenübersteht, als ein Feind, der aktiv angreift und einen vernichtenden Kampf gegen den erkrankten Menschen führt. Gleichzeitig wird die Depression als ein Stück Lebensweg, als ein Lebensabschnitt erlebt, der bewältigt werden muss.

Die diskursgrammatischen Analysen zeigen exemplarisch den Zusammenhang zwischen sprachlichen Oberflächenphänomenen, dem im Diskurs verhandelten Wissen und dessen Relevanz für die öffentlichen Diskurse. Die Konstruktionen im Sinne der KxG ermöglichen dabei einen guten Analysezugriff. Zudem zeigen die Analysen, dass psychische Krankheiten wie die Depression einen hohen Nachrichtenwert haben. 


\section{Literatur}

Antony, Laurence (2014): AntConc (Version 3.4.4.w) [Computer Software]. Tokyo: Waseda University. http://www.laurenceanthony.net/.

Bandura, Albert (2000): Exercise on Human Agency Through Collective Efficacy. Current Directions in Psychological Science 9 (3), 75-78.

Bentele, Günter, Hans-Bernd Brosius \& Otfried Jarren (2013): Lexikon Kommunikations- und Medienwissenschaft. Wiesbaden: Springer Fachmedien.

Brinker, Klaus (2010): Linguistische Textanalyse. Eine Einführung in Grundbegriffe und Methoden.7. Aufl. Berlin: Erich Schmidt.

Brünner, Gisela (2011): Gesundheit durchs Fernsehen. Linguistische Untersuchungen zur Vermittlung medizinischen Wissens und Aufklärung in Gesundheitsfragen. Duisburg: Rhein-Ruhr.

Bubenhofer, Noah (2018): Serialität der Singularität. Korpusanalyse narrativer Muster in Geburtsberichten. Zeitschrift für Literaturwissenschaft und Linguistik 48, 357-388.

Bücker, Jörg, Susanne Günthner \& Wolfgang Imo (2015): Einleitung. In Jörg Bücker, Susanne Günthner \& Wolfgang Imo (Hrsg.), Konstruktionsgrammatik V: Konstruktionen im Spannungsfeld von sequenziellen Mustern, kommunikativen Gattungen und Textsorten, 1-14. Tübingen: Stauffenburg.

Busch, Albert (1999): Semantische Vertikalität und diskursive Grundkonzepte in der Gesundheitskommunikation. In Jürg Niederhauser \& Kirsten Adamzik (Hrsg.), Wissenschaftssprache und Umgangssprache in Kontakt, 103-122. Frankfurt a.M.: Peter Lang.

Busch, Albert (2015): Medizindiskurse: Mediale Räume der Experten-Laien-Kommunikation. In Albert Busch \& Thomas Spranz-Fogasy (Hrsg.), Handbuch Sprache in der Medizin, 369-386. Berlin, Boston: De Gruyter.

Busse, Dietrich (1996): Öffentlichkeit als Raum für Diskurse. Entfaltungsbedingungen für Bedeutungswandel im öffentlichen Sprachgebrauch. In Karin Böke, Matthias Jung \& Martin Wengeler (Hrsg.), Öffentlicher Sprachgebrauch. Praktische, theoretische und historische Perspektiven. Georg Stötzel zum 60. Geburtstag gewidmet, 347-358. Opladen: Westdeutscher Verlag.

Busse, Dietrich \& Wolfgang Teubert (1994): Ist Diskurs ein sprachwissenschaftliches Objekt? In Dietrich Busse, Fritz Hermanns \& Wolfgang Teubert (Hrsg.), Begriffsgeschichte und Diskursgeschichte. Methodenfragen und Forschungsergebnisse der historischen Semantik, 10-18. Opladen: Westdeutscher Verlag.

Dijk, Teun Adrian van (2009): Discourse and Context. A Sociocognitive Approach. Cambridge: Cambridge University Press.

Ehrenberg, Alain (2013): Das erschöpfte Selbst. Depression und Gesellschaft in der Gegenwart. 7. Aufl. Frankfurt a.M.: Suhrkamp.

Feer, Hans (1987): Die Sprache der Psychiatrie. Eine linguistische Untersuchung. Berlin: Springer.

Felder, Ekkehard (2009): Sprache - das Tor zur Welt? Perspektiven und Tendenzen in sprachlichen Äußerungen. In Ekkehard Felder (Hrsg.), Sprache, 13-57. Berlin: Springer.

Felder, Ekkehard (2010): Semantische Kämpfe - Die Macht des deklarativen in Fachdiskursen. In Thomas Fuchs \& Grit Schwarzkopf (Hrsg.), Verantwortlichkeit - nur eine Illusion?, 13-59. Heidelberg: Winter. 
Felder, Ekkehard (2012): Pragma-semiotische Textarbeit und der hermeneutische Nutzen von Korpusanalysen für die linguistische Mediendiskursanalyse. In Ekkehard Felder, Marcus Müller \& Friedemann Vogel (Hrsg.), Korpuspragmatik. Thematische Korpora als Basis diskurslinguistischer Analysen, 115-174. Berlin, Boston: De Gruyter.

Felder, Ekkehard (2013): Faktizitätsherstellung mittels handlungsleitender Konzepte und agonaler Zentren. Der diskursive Wettkampf um Geltungsansprüche. In Ekkehard Felder (Hrsg.), Faktizitätsherstellung in Diskursen. Die Macht des Deklarativen, 13-28. Berlin, Boston: De Gruyter.

Foucault, Michel (1991): Die Ordnung des Diskurses. Mit einem Essay von Ralf Konersmann. Frankfurt a.M.: Suhrkamp.

Foucault, Michel (1997): Archäologie des Wissens. 8. Aufl. Frankfurt a.M.: Suhrkamp.

Galtung, Johan \& Mari Holmboe Ruge (1965): The Structure of the Foreign News. The Presentation of the Congo, Cuba and Cyprus Crises in Four Norwegian Newspapers. Journal of Peace Research 2, 64-91.

Gieber, Walter (1964): News is what Neswpapermen Make it. In Lewis Anthony Dexter \& David Manning White (Hrsg.), People, Society, and Mass Communications, 173-180. New York: Free Press of Glencoe.

Goldberg, Adele (2006): Constructions at Work. The Nature of Generalization in Language. Oxford: Oxford University Press.

Hampe, Beate (2017): Embodiment and Discourse: Dimensions and Dynamics of Contemporary Metaphor Theory. In Beate Hampe (Hrsg.), Metaphor. Embodied Cognition and Discourse, 3-23. Cambridge: Cambridge University Press.

Hartmann, Peter (1961): Zur Theorie der Sprachwissenschaft. Assen: van Gorcum

Heinemann, Wolfgang \& Dieter Viehweger (1991): Textlinguistik. Eine Einführung. Tübingen: Niemeyer.

lakushevich, Marina (2009): Stydno! Du sollst dich was schämen! Ein deutsch-russischer Sprachvergleich der Kosmetikwerbung. Frankfurt a.M.: Peter Lang.

lakushevich, Marina (2020a): „In der Seelenfinsternis gefangen“ Metaphern der Depression in den Deutschen Qualitätsmedien. Der Sprachdienst 64 (6), 260-272.

lakushevich, Marina (2020b): Konstruktionen und ihre Leistungen in massenmedialen Patho- und Salutodiskursen: Depression und Burnout. In Michel Lefèvre \& Katharina Mucha (Hrsg.), Konstruktionen, Kollokationen, Muster, 207-222. Tübingen: Stauffenburg.

Kickbusch, Ilona (2006): Die Gesundheitsgesellschaft. Hamburg: Verlag für Gesundheitsförderung.

Köller, Wilhelm (2004): Perspektivität und Sprache: zur Struktur von Objektivierungsformen in Bildern, im Denken und in der Sprache. Berlin, Boston: De Gruyter.

Kohring, Matthias (2005): Wissenschaftsjournalismus. Forschungsüberblick und Theorieentwurf. Konstanz: UVK.

Kühn, Peter (1995): Mehrfachadressierung. Tübingen: Niemeyer.

Labov, William \& Joshua Waletzky (1967): Narrative Analysis: Oral Versions of Personal Experience. In June Helm (Hrsg.), Essays on the Verbal and Visual Arts, 12-44. Seattle, London: University of Washington Press.

Lakoff, George \& Mark Johnson (1980): Metaphors We Live By. Chicago: University of Chicago Press.

Luhmann, Niklas (1975): Veränderungen im System gesellschaftlicher Kommunikation und die Massenmedien. In Oskar Schatz (Hrsg.), Die elektronische Revolution. Wie gefährlich sind die Massenmedien?, 13-30. Graz: Styria. 
Luhmann, Niklas (1988): Erkenntnis als Konstruktion. Bern: Bentelli.

Luhmann, Niklas (1995): Die Realität der Massenmedien. Opladen: Westdeutscher Verlag.

Müller, Marcus (2016): Diskursgrammatik als Grammatik indexikalischer Ordnungen. In Jianhua Zhu, Jin Zhao \& Michael Szurawitzki (Hrsg.), Akten des XIII. internationalen Germanistenkongresses Shanghai 2015, 303-307. Frankfurt a.M.: Peter Lang.

Pfeifer, Wolfgang (2012): Etymologisches Wörterbuch des Deutschen. Koblenz: Edition Kramer.

Polenz, Peter von (1988): Deutsche Satzsemantik. Grundbegriffe des Zwischen-den-ZeilenLesens. 2. Aufl. Berlin, New York: De Gruyter.

Rosengren, Karl Erik (1970): International News: Intra and Extra Media Date. Acta Sociologica 13, 96-109.

Schneider, Wolfgang \& Bernhard Strauß (2013): Medikalisierung. Psychotherapeut 58, 217-218.

Schulz, Winfried (1976): Die Konstruktion von Realität in den Nachrichtenmedien. Analyse der aktuellen Berichtserstattung. Freiburg, München: Karl Alber.

Schuster, Britt-Marie (2010): Auf dem Weg zur Fachsprache. Sprachliche Professionalisierung in der psychiatrischen Schreibpraxis (1800-1939). Berlin, New York: De Gruyter.

Sontag, Susan (1980): Krankheit als Metapher. 2. Aufl. München, Wien: Carl Hanser.

Spieß, Constanze (2011): Diskurshandlungen. Theorie und Methode linguistischer Diskursanalyse am Beispiel der Bioethikdebatte. Berlin, Boston: De Gruyter.

Spieß, Constanze (2014): Linguistische Metaphernanalyse. In Matthias Junge \& Anne-Katrin Hoklas (Hrsg.), Methoden der Metaphernforschung, 31-58. Wiesbaden: Springer.

Spitzmüller, Jürgen \& Ingo H. Warnke (2011): Diskurslinguistik. Eine Einführung in Theorien und Methoden der transtextuellen Sprachanalyse. Berlin, Boston: De Gruyter.

Stöckl, Hartmut (2004): Die Sprache im Bild - Das Bild in der Sprache. Zur Verknüpfung von Sprache und Bild im massenmedialen Text. Berlin, Boston: De Gruyter.

Vogt, Jochen (2014): Aspekte erzählender Prosa. Eine Einführung in Erzähltechnik und Romantheorie. 11. Aufl. München: Fink.

Teubert, Wolfgang (2019): Im Kopf oder im Diskurs: wo ist unsere Welt? Sprache und Denken. teksti i dyskurs - text und diskurs 12, 25-47.

Van Damme, Wim, Ritwik Dahake, Alexandre Delamou et al. (2020): The COVID-19 pandemic: diverse contexts; different epidemics - how and why? BMJ Global Health 5: e003098. doi: 10.1136/bmjgh-2020-003098.

Warnke, Ingo (2009): Die sprachliche Konstituierung von geteiltem Wissen in Diskursen. In Ekkehard Felder \& Marcus Müller (Hrsg.), Wissen durch Sprache. Theorie, Praxis und Erkenntnisinteresse des Forschungsnetzwerks „Sprache und Wissen “, 113-140. Berlin, Boston: De Gruyter.

Zifonun, Gisela, Ludger Hoffmann \& Bruno Strecker (Hrsg.) (1997): Grammatik der deutschen Sprache. 3 Bde. Berlin, New York: De Gruyter. 
